\title{
Female Urinary Incontinence: Prevalence, Risk Factors and Impact on the Quality of Life of Gynecological Clinic Attendees in Lagos, Nigeria
}

\author{
Fatimat Motunrayo Akinlusi, Tawaqualit Abimbola Ottun, Yusuf Abisowo Oshodi, Bilkees Oluwatoyin \\ Seriki, Folasade D. Haleemah Olalere, Taiwo Olufunmilayo Kuye
}

Department of Obstetrics and Gynecology, Lagos State University College of Medicine/ Lagos State University Teaching Hospital, Ikeja, Nigeria.

Received: November 28, 2019

Accepted: May 5, 2020

\begin{abstract}
Aims: To determine the prevalence of urinary incontinence, risk factors and impact on the quality of life in gynecological clinic attendees of a University Hospital.

Methods: A cross sectional descriptive study was conducted amongst gynecological clinic attendees in a Teaching Hospital in Nigeria from 1st February to 31st July 2017. Structured questionnaires were used to ascertain the presence of urinary incontinence. Socio-demographic and medical factors; impact on daily activities and treatment history were assessed. Women with and without urinary incontinence were compared. Univariate, bivariate and multivariable analyses were performed.
\end{abstract}

Results: There were 395 women of $25-67$ years (mean age $=38.81 \pm 10.1$ ). About $33 \%$ had experienced urinary incontinence in the previous 6 months with Urgency, Mixed and Stress urinary incontinence occurring in 18.0\%, 7.6\% and 7.3\% respectively. Independent risk factors for urinary incontinence were age (odds ratio $=0.49,95 \%$ confidence interval $[\mathrm{CI}]=0.26-0.92, \mathrm{P}=0.026$ ), higher body mass index (odds ratio $=1.92,95 \% \mathrm{CI}=1.53-3.00, \mathrm{P}=0.004$ ) and history of constipation (odds ratio $=2.11,95 \% \mathrm{CI}=1.30-3.43, \mathrm{P}=0.003$ ). About $47 \%$ of those with urinary incontinence admitted to negative feelings like anxiety and depression; $45 \%$ had moderate to severe impact on their quality of life in all domains but only $27.7 \%$ sought help.

Conclusions: Urinary incontinence is common and risk factors include older age, high body mass index and constipation. Despite its substantial impact on the quality of life, majority do not seek help. Addressing modifiable risks factors and improving treatment seeking behaviour will assist in reducing the prevalence of urinary incontinence.

Keywords: female urinary incontinence; quality of life; risk factors; stress incontinence; urgency incontinence.

Citation : Akinlusi FM, Ottun TA, Oshodi YA, Seriki BO, Olalere FDH, Kuye TO. Female Urinary Incontinence: Prevalence, Risk Factors and Impact on the Quality of Life of Gynecological Clinic Attendees in Lagos, Nigeria. Nep J Obstet Gynecol. 2020;15(30):3138. DOI: $10.3126 /$ njog.v15i1.29338

\section{INTRODUCTION}

Urinary incontinence (UI) remains a public health concern being increasingly reported across agegroups and socio-economic strata. It is estimated to affect 200 million people worldwide, majority of who are females. ${ }^{1}$ A systematic review reported a wide prevalence range of $16.2 \%-81.9 \% ;^{2}$ possibly a result of variations in study populations, definition of UI, utilization of different sampling techniques and survey tools.

Though not often reported, leakage of urine is experienced by many women, who are approximately three times more likely than men to have urinary

\section{CORRESPONDENCE}

Fatimat Motunrayo Akinlusi

Consultant Obstetrician/ Gynecologist

Lagos State University College of Medicine, Ikeja, Nigeria

Email: fatimatakinlusi@yahoo.co.uk; Phone: +234-8034069207 incontinence. ${ }^{3}$ Stress UI however dominates in younger women while urge and mixed incontinence increase with age.

Age, obstetric factors, hysterectomy and obesity are the most common risk factors identified by epidemiological studies. ${ }^{46}$ Smoking, menstrual cycle, menopause, fluid intake, constipation, and racial differences are however less often reported. ${ }^{7}$

Urinary incontinence impacts considerably on the quality of life, causing disability, embarrassment, social isolation, avoidance of sexual activity and dependence even among healthy looking women. 
It may be associated with physical/psychological distress within the incontinent female population. ${ }^{8}$ Apart from individual sufferings; UI causes substantial economic impact on the health systems accounting for a notable proportion of the total health-care budget in certain climes. ${ }^{9}$

Despite its impacts on the quality of life, UI has not received adequate attention in Nigeria. This study sets out to determine the prevalence of urinary incontinence; associated risk factors; impact on the quality of life and health seeking behavior of gynecological clinic attendees in a Teaching Hospital in Nigeria.

\section{METHODS}

A cross-sectional descriptive study conducted at the gynecological clinic of a University Teaching Hospital between 1st February and 31st July, 2017.

All consecutive attendees at the gynecological clinic during the study period were invited to participate irrespective of their age, parity or reason for consultation. Only non-consenting and mentally unfit women were excluded.

Pretested structured questionnaires were administered by trained research assistants to determine the presence of UI which was defined as complaint of any involuntary leakage of urine ${ }^{10}$ in the previous six months. The severity of incontinence was also evaluated by the frequency and duration of urine leakage, as described by the women. Additional data obtained were socio-demographic characteristics; obstetrics, gynecological, medical and surgical risk factors; treatment history as well as impact on daily activities and quality of life (QOL). Women with and without urinary incontinence were compared.

A total of eleven questions were used to assess the impact of urinary incontinence on the quality of life of participants. The impact level on daily activities was measured on a Likert scale categorized into 'never affected', 'little affected', 'somewhat', 'much' and 'a great deal' which corresponded to scores of
$0,1,2,3$ and 4 respectively. Possible scores ranged from 0 to 44 with higher scores reflecting increased impact on the QOL. Scores of $0-14 ; 15-29$; and 30 or more; were categorized as mild, moderate and severe impact.

SPSS version 22.0 (statistical package for social sciences Inc. Chicago, III) was used for data analyses. Descriptive analysis as well as Pearson's Chi-squared test was used with p-value of 0.05. Multivariable logistic regression was used to identify factors that increased the odds of urinary incontinence.

Informed consent was obtained and approval for the study was from the hospital ethical review committee.

\section{RESULTS}

A total of 395 women between age 25-67 years (mean age $=38.81 \pm 10.1$ ) were enrolled; $61.3 \%$ had tertiary education and $50 \%$ were either overweight or obese. Single women were more $(54.7 \%)$ than married (38.5\%) and almost half of the women were primiparous. Nine in ten respondents had only vaginal deliveries (spontaneous or instrumental). Though greater than $90 \%$ of respondents received antenatal and postnatal care; just about $10 \%$ reported learning perineal exercises during pregnancy while $4 \%$ practiced these exercises after pregnancy. Only $10.6 \%$ of women had one or more caesarean sections till the time of this study. Forty percent had a history of constipation, $19.7 \%$ had a previous history of urinary tract infection while $29.4 \%$ had a history of dilatation and curettage.

Of the 395 participants enrolled in the study, 130 had experienced UI in the previous 6 months giving a prevalence rate of $32.9 \%$. Urgency, Mixed and Stress UI occurred in $71(18.0 \%), 30(7.6 \%)$ and $29(7.3 \%)$ respectively.

Age and body mass index (BMI) were potentially associated with urinary incontinence in our bivariate analysis. Women without urinary incontinence were younger than women with incontinence and the age difference was statistically significant [Table-1]. 
Female incontinence: Prevalence, Risk Factors, QOL

Table-1: Urinary Incontinence and Socio-demographic Characteristics

\begin{tabular}{|c|c|c|c|c|c|}
\hline \multirow{2}{*}{ Variable } & \multicolumn{2}{|c|}{ Urinary incontinence } & \multirow{2}{*}{ Total } & \multirow{2}{*}{$\mathbf{X}^{2}$} & \multirow{2}{*}{ p-value } \\
\hline & Yes $(n=130)$ & No $(n=265)$ & & & \\
\hline $\begin{array}{l}\text { Age-group (years } \\
\leq 30 \\
31-40 \\
41-50 \\
>50\end{array}$ & $\begin{array}{l}29(40.8) \\
49(25.7) \\
37(38.5) \\
15(40.5)\end{array}$ & $\begin{array}{c}42(59.2) \\
142(74.3) \\
59(61.5) \\
22(59.5)\end{array}$ & $\begin{array}{c}71(100.0) \\
191(100.0) \\
96(100.0) \\
37(100.0)\end{array}$ & 8.933 & $0.030^{*}$ \\
\hline Mean Age \pm SD & $43.37 \pm 10.9$ & $38.53 \pm 9.7$ & & & $0.025^{*}$ \\
\hline $\begin{array}{l}\text { Marital status } \\
\text { Single } \\
\text { Married } \\
\text { Divorced } \\
\text { Widowed }\end{array}$ & $\begin{array}{c}67(31.0) \\
53(34.9) \\
7(43.8) \\
3(27.3)\end{array}$ & $\begin{array}{c}149(69.0) \\
99(65.1) \\
9(56.2) \\
8(72.7)\end{array}$ & $\begin{array}{c}216(100.0) \\
152(100.0) \\
16(100.0) \\
11(100.0)\end{array}$ & 1.624 & 0.654 \\
\hline $\begin{array}{l}\text { Educational level } \\
\text { None } \\
\text { Primary } \\
\text { Secondary } \\
\text { Tertiary }\end{array}$ & $\begin{array}{l}10(47.6) \\
15(37.5) \\
34(37.0) \\
71(29.3)\end{array}$ & $\begin{array}{c}11(52.4) \\
25(62.5) \\
58(63.0) \\
171(70.7)\end{array}$ & $\begin{array}{c}21(100.0) \\
40(100.0) \\
92(100.0) \\
242(100.0)\end{array}$ & 4.519 & 0.211 \\
\hline $\begin{array}{l}\text { Occupation } \\
\text { Civil servant } \\
\text { Skilled worker } \\
\text { Semi-skilled } \\
\text { Petty trader } \\
\text { unemployed }\end{array}$ & $\begin{array}{c}35(29.4) \\
34(34.3) \\
5(29.4) \\
45(36.0) \\
11(31.4)\end{array}$ & $\begin{array}{l}84(70.4) \\
65(65.7) \\
12(70.6) \\
80(64.0) \\
24(68.6)\end{array}$ & $\begin{array}{c}119(100.0) \\
99(100.0) \\
17(100.0) \\
125(100.0) \\
35(100.0)\end{array}$ & 1.421 & 0.840 \\
\hline $\begin{array}{l}\text { BMI class } \\
\text { Underweight } \\
\text { Normal } \\
\text { Overweight } \\
\text { Obese }\end{array}$ & $\begin{array}{c}7(87.5) \\
70(37.8) \\
26(22.2) \\
27(31.8)\end{array}$ & $\begin{array}{c}1(12.5) \\
115(62.2) \\
91(77.8) \\
58(68.3)\end{array}$ & $\begin{array}{c}8(100.0) \\
185(100.0) \\
117(100.0) \\
85(100.0)\end{array}$ & 18.936 & $0.001^{*}$ \\
\hline
\end{tabular}

Majorities (87.6\%) were premenopausal. Parity, learning perineal exercises during antenatal period and postpartum practice of pelvic exercises were significantly associated with urinary incontinence in our bivariate analysis. Interestingly menopausal status was not associated with urinary incontinence. Not a single one of the 31 nulliparous women, had experienced UI whereas more than $50 \%$ of the multiparous women had experienced $\mathrm{UI}$ in the previous 6 months [Table-2].

Table-2: Urinary Incontinence and Reproductive History

\begin{tabular}{|c|c|c|c|c|c|}
\hline \multirow{2}{*}{ Variable } & \multicolumn{2}{|c|}{ Urinary incontinence } & \multirow{2}{*}{ Total } & \multirow{2}{*}{$\mathbf{X}^{2}$} & \multirow{2}{*}{ p-value } \\
\hline & Yes $(n=130)$ & No $(n=265)$ & & & \\
\hline $\begin{array}{l}\text { Age at menarche } \\
<13 \\
13-17 \\
>17\end{array}$ & $\begin{array}{l}21(32.8) \\
96(32.1) \\
13(40.6)\end{array}$ & $\begin{array}{c}43(67.2) \\
203(67.9) \\
19(59.4)\end{array}$ & $\begin{array}{c}64(100.0) \\
299(100.0) \\
32(100.0\end{array}$ & 0.950 & 0.622 \\
\hline $\begin{array}{l}\text { Age at marriage }(\mathrm{n}=178) \\
<18 \\
18-35 \\
>35\end{array}$ & $\begin{array}{c}5(45.5) \\
44(31.4) \\
13(48.1)\end{array}$ & $\begin{array}{c}6(54.5) \\
96(68.6) \\
14(51.9)\end{array}$ & $\begin{array}{c}11(100.0) \\
140(100.0) \\
27(100.0)\end{array}$ & 3.370 & 0.185 \\
\hline $\begin{array}{l}\text { Menopausal status } \\
\text { Yes } \\
\text { No }\end{array}$ & $\begin{array}{c}22(44.9) \\
108(31.2)\end{array}$ & $\begin{array}{c}27(55.1) \\
238(68.8)\end{array}$ & $\begin{array}{c}49(100.0) \\
346(100.0)\end{array}$ & 3.460 & 0.056 \\
\hline $\begin{array}{l}\text { Parity } \\
0 \\
1 \\
2 \\
3 \\
\geq 4\end{array}$ & $\begin{array}{c}0(0.0) \\
39(20.6) \\
29(41.4) \\
42(61.8) \\
20(54.1)\end{array}$ & $\begin{array}{c}31(100.0) \\
150(100.0) \\
41(100.0) \\
26(100.0) \\
17(100.0)\end{array}$ & $\begin{array}{c}31(100.0) \\
189(100.0) \\
70(100.0) \\
68(100.0) \\
37(100.0)\end{array}$ & 63.538 & $0.001 *$ \\
\hline
\end{tabular}




\begin{tabular}{|c|c|c|c|c|c|}
\hline \multirow{2}{*}{ Variable } & \multicolumn{2}{|c|}{ Urinary incontinence } & \multirow{2}{*}{ Total } & \multirow{2}{*}{$\mathbf{X}^{2}$} & \multirow{2}{*}{ p-value } \\
\hline & Yes $(n=130)$ & No $(n=265)$ & & & \\
\hline $\begin{array}{l}\text { Age at delivery } \\
\leq 18 \\
>18\end{array}$ & $\begin{array}{c}9(36.0) \\
121(32.7)\end{array}$ & $\begin{array}{c}16(64.0) \\
249(100.0)\end{array}$ & $\begin{array}{c}25(100.0) \\
370(100.0)\end{array}$ & 0.115 & 0.737 \\
\hline $\begin{array}{l}\text { Delivery type } \\
\text { Vaginal } \\
\text { Caesarean section }\end{array}$ & $\begin{array}{c}115(32.6) \\
15(35.7)\end{array}$ & $\begin{array}{c}238(67.4) \\
27(64.3)\end{array}$ & $\begin{array}{l}353(100.0 \\
42(100.0)\end{array}$ & 0.167 & 0.683 \\
\hline $\begin{array}{l}\text { Labour duration (hours) } \\
<12 \\
\geq 12\end{array}$ & $\begin{array}{c}112(34.8) \\
18(24.7)\end{array}$ & $\begin{array}{c}210(65.2) \\
55(75.3)\end{array}$ & $\begin{array}{c}322(100.0) \\
73(100.0)\end{array}$ & 2.763 & 0.096 \\
\hline $\begin{array}{l}\text { Birth weight (gram) } \\
\leq 3500 \\
>3500\end{array}$ & $\begin{array}{c}112(32.5) \\
18(36.0)\end{array}$ & $\begin{array}{c}233(67.5) \\
32(64.0)\end{array}$ & $\begin{array}{c}345(100.0) \\
50(100.0)\end{array}$ & 0.247 & 0.619 \\
\hline $\begin{array}{l}\text { Antenatal care } \\
\text { Yes } \\
\text { No }\end{array}$ & $\begin{array}{c}129(33.5) \\
1(10.0)\end{array}$ & $\begin{array}{c}256(66.5) \\
9(90.0)\end{array}$ & $\begin{array}{c}385(100.0) \\
10(100.0)\end{array}$ & 2.439 & 0.118 \\
\hline $\begin{array}{l}\text { Learnt perineal exercise during } \mathrm{ANC} \\
\text { Yes } \\
\text { No }\end{array}$ & $\begin{array}{c}22(52.4) \\
108(30.6)\end{array}$ & $\begin{array}{c}20(47.6) \\
245(69.4)\end{array}$ & $\begin{array}{c}42(100.0) \\
353(100.0)\end{array}$ & 8.068 & $0.005^{*}$ \\
\hline $\begin{array}{l}\text { Practiced pelvic exercise during postnatal period } \\
\text { Yes } \\
\text { No }\end{array}$ & $\begin{array}{c}10(66.7) \\
120(31.6)\end{array}$ & $\begin{array}{c}5(33.3) \\
260(68.4)\end{array}$ & $\begin{array}{c}15(100.0) \\
380(100.0)\end{array}$ & 8.046 & $0.003 *$ \\
\hline $\begin{array}{l}\text { Postnatal care } \\
\text { Yes } \\
\text { No }\end{array}$ & $\begin{array}{c}125(33.6) \\
5(21.7)\end{array}$ & $\begin{array}{c}246(66.4) \\
18(78.3)\end{array}$ & $\begin{array}{c}372(100.0) \\
23(100.0)\end{array}$ & 1.381 & 0.240 \\
\hline $\begin{array}{l}\text { Immediate postnatal complication } \\
\text { Yes } \\
\text { No }\end{array}$ & $\begin{array}{c}5(45.5) \\
125(32.6)\end{array}$ & $\begin{array}{c}6(54.5) \\
259(67.4)\end{array}$ & $\begin{array}{c}11(100.0) \\
384(100.0)\end{array}$ & 0.806 & 0.369 \\
\hline
\end{tabular}

Of the past medical indices assessed, previous constipation, history of urinary tract infection (UTI), previous pelvic inflammatory disease (PID) and past dilation and curettage (D\&C) were similarly associated with urinary incontinence in the bivariate analysis. A half of the 78 women with positive history of UTI experienced UI while just about a quarter (28.7\%) of the 317 women without UTI admitted to previous urinary incontinence [Table-3].

Table-3: Association between Urinary Incontinence and Past Medical History

\begin{tabular}{|c|c|c|c|c|c|}
\hline \multirow{2}{*}{ Variable } & \multicolumn{2}{|c|}{ Urinary incontinence } & \multirow{2}{*}{ Total } & \multirow{2}{*}{$\mathbf{X}^{2}$} & \multirow{2}{*}{ p-value } \\
\hline & Yes $(n=130)$ & No $(n=265)$ & & & \\
\hline $\begin{array}{l}\text { Previous constipation } \\
\text { Yes } \\
\text { No }\end{array}$ & $\begin{array}{l}71(44.9) \\
59(24.9)\end{array}$ & $\begin{array}{c}87(55.1) \\
178(75.1)\end{array}$ & $\begin{array}{l}158(100.0) \\
237(100.0)\end{array}$ & 17.247 & $0.001^{*}$ \\
\hline $\begin{array}{l}\text { Previous cough }>8 \text { weeks } \\
\text { Yes } \\
\text { No }\end{array}$ & $\begin{array}{r}11(45.8) \\
119(32.1)\end{array}$ & $\begin{array}{c}13(54.2) \\
252(67.9)\end{array}$ & $\begin{array}{c}24(100.0) \\
371(100.0)\end{array}$ & 1.932 & 0.164 \\
\hline $\begin{array}{l}\text { Previous pelvic surgery } \\
\text { Yes } \\
\text { No }\end{array}$ & $\begin{array}{c}30(36.1) \\
100(32.1)\end{array}$ & $\begin{array}{c}53(63.9) \\
212(67.9)\end{array}$ & $\begin{array}{c}83(100.0) \\
312(100.0)\end{array}$ & 0.497 & 0.481 \\
\hline $\begin{array}{l}\text { History of UTI in the past } \\
\text { Yes } \\
\text { No }\end{array}$ & $\begin{array}{l}39(50.0) \\
91(28.7)\end{array}$ & $\begin{array}{c}39(50.0) \\
226(71.3)\end{array}$ & $\begin{array}{c}78(100.0) \\
317(100.0)\end{array}$ & 12.854 & $0.001^{*}$ \\
\hline $\begin{array}{l}\text { History of PID } \\
\text { Yes } \\
\text { No }\end{array}$ & $\begin{array}{l}47(44.3) \\
83(28.7)\end{array}$ & $\begin{array}{c}59(55.7) \\
206(71.3)\end{array}$ & $\begin{array}{l}106(100.0) \\
289(100.0)\end{array}$ & 8.570 & $0.003 *$ \\
\hline $\begin{array}{l}\text { Past dilation \&curettage } \\
\text { Yes } \\
\text { No }\end{array}$ & $\begin{array}{l}49(42.2) \\
81(29.0)\end{array}$ & $\begin{array}{r}67(57.8) \\
198(71.0)\end{array}$ & $\begin{array}{l}116(100.0) \\
279(100.0)\end{array}$ & 6.475 & $0.011^{*}$ \\
\hline
\end{tabular}


On Logistic Regression analysis, independent risk factors for UI were age (odds ratio $=0.49,95 \%$ confidence interval $[\mathrm{CI}]=0.26-0.92, \mathrm{P}=0.026)$, higher body mass index (odds ratio $=1.92,95 \% \mathrm{CI}=1.53-3.00, \mathrm{P}=0.004)$ and history of constipation (odds ratio $=2.11,95 \% \mathrm{CI}=1.30-3.43, \mathrm{P}=0.003$ ). Women between 31 and 40 years were less likely than those aged 30 years and below to experience UI. Also, overweight and obese women were at least one and a half times more likely to experience UI than women with normal weight. Those with positive history of constipation had two times the risk of UI than those without constipation [Table-4].

Table-4: Logistic regression showing independent predictors for urinary incontinence

\begin{tabular}{|c|c|c|c|}
\hline Variable & p-value & Odds ratio & $95 \% \mathrm{CI}$ \\
\hline $\begin{array}{l}\text { Age group (Years) } \\
\leq 30 \\
31-40 \\
41-50 \\
>50\end{array}$ & $\begin{array}{c}0.026^{*} \\
0.374 \\
0.759\end{array}$ & $\begin{array}{c}1 \\
0.485 \\
0.721 \\
0.860\end{array}$ & $\begin{array}{l}0.256-0.919 \\
0.350-1.483 \\
0.329-2.249\end{array}$ \\
\hline $\begin{array}{l}\text { BMI class } \\
\text { Normal } \\
\text { Underweight } \\
\text { Overweight } \\
\text { Obese }\end{array}$ & $\begin{array}{c}0.059 \\
0.019^{*} \\
0.004^{*}\end{array}$ & $\begin{array}{c}1 \\
1.332 \\
1.552 \\
1.918\end{array}$ & $\begin{array}{l}0.709-1.854 \\
1.345-2.342 \\
1.532-3.004\end{array}$ \\
\hline $\begin{array}{l}\text { Learnt perineal exercise during antenatal or postnatal } \\
\text { period } \\
\text { No } \\
\text { Yes }\end{array}$ & 0.076 & $\begin{array}{c}1 \\
1.939\end{array}$ & $0.932-4.035$ \\
\hline $\begin{array}{l}\text { Practised pelvic exercise in postnatal period } \\
\text { No } \\
\text { Yes }\end{array}$ & 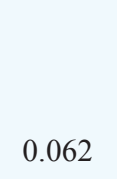 & $\begin{array}{c}1 \\
3.313\end{array}$ & $0.940-11.673$ \\
\hline $\begin{array}{l}\text { Number of pregnancy } \\
<2 \\
>2\end{array}$ & 0.860 & $\begin{array}{c}1 \\
1.063\end{array}$ & $0.542-2.081$ \\
\hline $\begin{array}{l}\text { Previous constipation } \\
\text { No } \\
\text { Yes }\end{array}$ & $0.003 *$ & $\begin{array}{c}1 \\
2.111\end{array}$ & $1.299-3.430$ \\
\hline $\begin{array}{l}\text { Previous history of UTI } \\
\text { No } \\
\text { Yes }\end{array}$ & 0.056 & $\begin{array}{c}1 \\
1.738\end{array}$ & $0.985-3.066$ \\
\hline $\begin{array}{l}\text { History of PID } \\
\text { No } \\
\text { Yes }\end{array}$ & 0290 & $\begin{array}{c}1 \\
1.344\end{array}$ & $0.777-2.325$ \\
\hline $\begin{array}{l}\text { History of dilation and curettage } \\
\text { No } \\
\text { Yes }\end{array}$ & 0.179 & $\begin{array}{c}1 \\
1.420\end{array}$ & $0.851-2.371$ \\
\hline
\end{tabular}

Among patients with urinary incontinence $45 \%$ have moderate to severe affectation of their quality of life in all domains. About $47 \%$ of those with UI admitted to notable negative feelings like anxiety and depression [Table-5]. 
Table-5: Quality of life of respondents with Urinary incontinence

\begin{tabular}{|l|l|l|l|l|l|}
\hline Extent to which the condition affects respondents $(\mathbf{n}=\mathbf{1 3 0})$ & Never & Little & Somewhat & Much & A great deal \\
\hline Prevented performance of domestic activity & $63(48.5)$ & $11(8.5)$ & $52(40.0)$ & $4(3.1)$ & $0(0.0)$ \\
\hline Prevented occupational engagement & $72(55.4)$ & $7(5.4)$ & $49(37.7)$ & $2(1.5)$ & $0(0.0)$ \\
\hline Prevented long distance travels & $63(48.5)$ & $7(5.4)$ & $55(42.3)$ & $5(3.8)$ & $0(0.0)$ \\
\hline Prevented interaction with other people & $77(59.2)$ & $1(0.8)$ & $51(39.2)$ & $0(0.0)$ & $1(0.8)$ \\
\hline Prevented attendance at religious ceremonies and other functions & $68(52.3)$ & $6(4.6)$ & $52(40.0)$ & $2(1.5)$ & $2(1.5)$ \\
\hline $\begin{array}{l}\text { Prevented leisure activities } \\
\text { Caused reduction in sexual activity }\end{array}$ & $62(47.7)$ & $5(3.8)$ & $58(44.6)$ & $3(2.3)$ & $2(1.5)$ \\
\hline Reduction in sexual satisfaction & $50(38.5)$ & $9(6.9)$ & $60(46.2)$ & $4(3.1)$ & $7(5.4)$ \\
\hline Invoked fear of rejection by partner & $55(42.3)$ & $10(7.7)$ & $54(41.5)$ & $6(4.6)$ & $5(3.8)$ \\
\hline Caused additional financial burden through medical care or laundry & $58(44.6)$ & $4(3.1)$ & $57(43.9)$ & $4(3.1)$ & $7(5.4)$ \\
\hline $\begin{array}{l}\text { Invoked negative feeling such as blue mood, despair, anxiety and } \\
\text { depression }\end{array}$ & $51(39.2)$ & $5(3.9)$ & $61(46.9)$ & $10(7.7)$ & $3(2.3)$ \\
\hline
\end{tabular}

Only about a quarter of respondents sought help for UI. Of these, $91.7 \%$ sought orthodox medical interventions at health care facilities, while the rest reported use of alternative therapy. Majority however claimed there was no improvement in their condition even after intervention [Table-6].

Table-6: Treatment seeking behavior of respondents with urinary incontinence

\begin{tabular}{|l|l|l|}
\hline \multicolumn{1}{|c|}{ Variable } & $\begin{array}{c}\text { Frequency } \\
(\mathbf{n = 1 3 0 )}\end{array}$ & $\begin{array}{l}\text { Percentage } \\
(\%)\end{array}$ \\
\hline $\begin{array}{l}\text { Previous history of seeking } \\
\text { treatment }\end{array}$ & & \\
$\begin{array}{l}\text { Yes } \\
\text { No }\end{array}$ & 36 & 27.7 \\
\hline $\begin{array}{l}\text { Place of treatment }(\mathrm{n}=36) \\
\text { Hospital } \\
\text { Traditional healer }\end{array}$ & 94 & 72.3 \\
\hline $\begin{array}{l}\text { Type of treatment }(\mathrm{n}=36) \\
\text { Pelvic floor exercise }\end{array}$ & 33 & 91.7 \\
Medication & 9 & 8.3 \\
Surgery & 24 & 25.0 \\
\hline $\begin{array}{l}\text { Effectiveness of treatment } \\
\text { Effective }\end{array}$ & 3 & 66.7 \\
Not effective & 13 & 8.3 \\
Minimal improvement & 19 & 36.1 \\
\hline
\end{tabular}

\section{DISCUSSION}

The prevalence of urinary incontinence in this study was $32.9 \%$, which is comparable with $30.6 \%$, $29 \%$ and $38.1 \%$ previously reported. ${ }^{11-13}$ but much higher than $2.8 \%, 5.2 \%, 12.2 \%$ and $15.2 \%$ documented by Ojengbede, Badejoko, Obioha and
Rabiu respectively. ${ }^{14-17}$ The ICS definition of UI was adopted in our study as in the EPINCONT study ${ }^{12}$ and both reported comparable prevalence. Though majority of our participants were less than 50 years, similar to a Nigerian survey, ${ }^{14}$ our prevalence is much higher, possibly a result of the hospital based nature and the fact that current UI was not reported separately from previous UI in our study.

Variations in prevalence may be due to differences in definitions, study type, target, population, inaccuracies of self-reporting and interval between studies amongst others.

Age group 31-40 years recorded the highest number of UI, similar to findings in Ilorin and Uyo. ${ }^{11,13}$ Incidence of UI was also found to be highest in women 20 39 years of age in the Norwegian EPINCONT study.

Though stress urinary incontinence is predominant amongst younger women according to reports in most literature, 11,12,18 urgency incontinence was the most prevalent in our study. This is contrary to our expectations but similar to some other study findings. ${ }^{15,19,20}$ This may be related to the reported history of urinary tract infection (UTI) in about 20\% of our study population. UTI is a treatable cause of UI. It will be interesting to investigate this in more details in the near future.

Overweight, ${ }^{5} \mathrm{BMI},{ }^{12}$ age, ${ }^{16}$ and parity ${ }^{11,12}$ have been previously established to be associated with urinary 
incontinence. Ojengbede et al ${ }^{14}$ found age, number of children, location, delivery mode and history of diabetes to be significantly associated with urge incontinence; however, only location remained significant in the logistic model.

We also found significant association of UI with age, body mass index (BMI), practice of postnatal pelvic exercise, parity, constipation, UTI, PID, dilatation and curettage. However, the independent risk factors were age, BMI and constipation.

Combined analyses of the incontinence types in our study might have contributed to the differences in independent risk factors documented. Our findings are similar to those of Ijaiya, ${ }^{11}$ who found significant association of these factors with urinary incontinence.

Epidemiological studies cite overweight and obesity as important risk factors for urinary incontinence. ${ }^{5}$ Weight loss by both surgical and more conservative approaches is effective in reducing urinary incontinence symptoms and should be strongly considered as a first line treatment for overweight and obese women with urinary incontinence. ${ }^{5}$ With advancing age and obesity there is progressive loss of muscle tone, decreased contractility and changes in hormonal stimulation.

Pregnancy and labor are known to predispose to a combination of injury to the pelvic floor musculature, connective tissue and nerves. Marinkovic and his colleagues $^{21}$ emphasized that trauma to the pelvic floor muscles and structures from childbirth could result in stress urinary incontinence. It is instructive to note that our study revealed no independent association with parity.
In all the domains assessed such as social interaction, sexual activity, finances and emotional status, quality of life was impacted significantly. About $47 \%$ of those with UI admitted to having negative feelings such as despair, anxiety and depression while 45\% had a cumulative moderate to severe impact on their quality of life in all domains.

Respondents' health seeking behavior was poor with merely $27.7 \%$ of those affected seeking care. This is comparable with findings in Spain, Germany, France, and Pennsylvania where $24 \%, 25 \%, 33 \%$ and $25.5 \%$ sought help ${ }^{22,23}$ but higher than $12.9 \%$ reported in Ibadan. $^{24}$

Women may not seek help if they perceive UI as a usual, unavoidable part of aging not warranting hospital consultation. Others may not, possibly due to embarrassment or lack of knowledge about availability of treatment ${ }^{25}$ The severity of UI may also affect the health care seeking behavior. Mild UI might contribute to deferral of treatment. Meral Kılıç, in his study, reported mild urinary incontinence in $77.5 \%$ of incontinent participants and only $29.3 \%$ of them sought help. ${ }^{8}$ He concluded that the low rate may be because majority of the women had mild urinary incontinence.

\section{CONCLUSIONS}

Urinary incontinence is a common problem amongst reproductive age women and most do not seek help. Independent risk factors include age, body mass index, and history of constipation. It impacts significantly on women's quality of life. Women should be encouraged to avoid modifiable risk factors and disclose urinary leakage promptly. This will enhance early intervention and better quality of life.

\section{REFERENCES}

1. Taylor W, Weir M, Cahill J, Rizk D. The Self-reported Prevalence and Knowledge of Urinary Incontinence and Barriers to Health Care-Seeking in a Community Sample of Canadian Women. Am J Med Sci. 2013;3(5):97-102

2. Kwon BE, Kim GY, Son YJ, Roh YS, You MA. Quality of life of women with urinary incontinence: A systematic literature review. Int Neurourol J. 2010;14(3):133-8. doi:10.5213/ inj.2010.14.3.133.

3. Milsom I, Gyhagen M. The prevalence of urinary incontinence. Climacteric. 2019;22(3):217-22. doi:10.1080/136971 37.2018.1543263.

4. Sangsawang B, Sangsawang N. Stress urinary incontinence in pregnant women: A review of prevalence, pathophysiology, and treatment. Int Urogynecol J. 2013;24(6):901-12. doi:10.1007/s00192-013-2061-7.

5. Whitcomb EL, Subak LL. Effect of weight loss on urinary incontinence in women. Open Access J Urol. 2011;3(1):123-32. doi:10.2147/OAJU.S21091

6. Faiena I, Patel N, Parihar JS, Calabrese M, Tunuguntla H, Robert R. Conservative Management of Urinary Incontinence in Women. Rev Urol. 2015;17(3):129-39. doi:10.3909/ riu0651.

7. Cameron AP, Heidelbaugh JJ, Jimbo M. Diagnosis and office-based treatment of urinary incontinence in adults. Part 
one: Diagnosis and testing. Ther Adv Urol. 2013;5(4):181-7. doi: $10.1177 / 1756287213489720$.

8. Kilıc M. Incidence and risk factors of urinary incontinence in women visiting Family Health Centers. Springerplus. 2016;5(1). doi:10.1186/s40064-016-2965-z.

9. Wagner T, Moore K, Subak L, De Wachter S, Dudding T. Incontinence : 6th ICI. 6th ed. (Abrams P, Cardozo L, Wagg A, Wein A, eds.). Tokyo; 2016. https://www.ics.org/education/ icspublications/icibooks/6thicibook\%0Ahttps://www.ics.org/ education/icspublications/icibooks.

10. Haylen BT, Ridder D de, Freeman RM, et al. An International Urogynecological Association (IUGA)/Internationa Continence Society (ICS) Joint Report on the Terminology for Female Pelvic Floor Dysfunction. Neurourol Urodyn. 2010;29:4-20. doi:10.1002/nau.

11. Ijaiya MA, Raji HO, Aboyeji AP, Adesina KT, Adebara IO, Ezeoke GG. Non-fistulous urinary leakage among women attending a Nigerian family planning clinic. Int J Womens Health. 2011;3:409-413. doi:10.2147/IJWH.S23179.

12. Ebbesen MH, Hunskaar S, Rortveit G, Hannestad YS. Prevalence, incidence and remission of urinary incontinence in women: Longitudinal data from the Norwegian HUNT study (EPINCONT). BMC Urol. 2013;13. doi:10.1186/1471-249013-27.

13. Udokang $\mathrm{N}$, Inyang $\mathrm{O}$, Dick $\mathrm{S}$. Urinary Incontinency in Women in Uyo Metropolis, South-South, Nigeria. Eur J Sustain Dev. 2016;5(1):145-52. doi:10.14207/ejsd.2016.v5n1p145.

14. Ojengbede OA, Morhason-Bello IO, Adedokun BO, Okonkwo NS, Kolade CO. Prevalence and the associated trigger factors of urinary incontinence among 5000 black women in sub-Saharan Africa: Findings from a community survey BJU Int. 2011;107(11):1793-1800. doi:10.1111/j.1464 410X.2010.09758.x.

15. Badejoko OO, Bola-Oyebamiji S, Awowole IO, Salako AA, Ogunniyi SO. Urinary incontinence: prevalence, pattern, and opportunistic screening in Ile-Ife, Nigeria. Int Urogynecol J. 2016;27(2):269-73. doi:10.1007/s00192-015-2826-2.

16. Obioha KC, Ugwu EO, Obi SN, Dim CC, Oguanuo TC. Prevalence and predictors of urinary/anal incontinence after vaginal delivery: prospective study of Nigerian women. Int
Urogynecol J. 2015;26(9):1347-54 doi:10.1007/s00192-0152690-0.

17. Press D. Risk factors for maternal mortality associated with eclampsia presenting at a Nigerian tertiary hospital. Int J Womens Health. 2018;10:715-21. doi:10.2147/IJWH.S178729.

18. Milsom I, Altman D, Cartwright R, Lapitan MC, Nelson R, Sillén U, et al. Epidemiology of Urinary Incontinence (UI) and other Lower Urinary Tract Symptoms (LUTS), Pelvic Organ Prolapse (POP) and Anal Incontinence (AI). In Abrams P, Cardozo L, Khoury S, Wein AJ, editors, Incontinence: 5th International Consultation on Incontinence, Paris, February 2012. 5th ed ed. Paris: ICUD-EAU. 2013. p. 15-107.

19. Agarwal BK, Agarwal N. Urinary incontinence: prevalence, risk factors, impact on quality of life and treatment seeking behaviour among middle aged women. Int Surg J. 2017;4(6):1953-8. doi:10.18203/2349-2902.isj20172131.

20. Sensoy N, Dogan N, Ozek B, Karaaslan L. Urinary incontinence in women: Prevalence rates, risk factors and impact on quality of life. Pak J Med Sci. 2013;29(3). doi:10.12669/ pjms.293.3404.

21. Marinkovic SP, Rovner ES, Moldwin RM, Stanton SL, Gillen LM, Marinkovic CM. The management of overactive bladder syndrome. BMJ. 2012;344(7853). doi:10.1136/bmj.e2365.

22. Junqueira JB, Santos VLC de G. Urinary incontinence in hospital patients: prevalence and associated factors. Rev Lat Am Enfermagem. 2018;25:e2970. doi:10.1590/1518 8345.2139 .2970

23. Pedersen SL, Lose G, Høybye MT, Elsner S, Waldmann A, Rudnicki M. Prevalence of urinary incontinence among women and analysis of potential risk factors in Germany and Denmark. Acta Obstet Gynecol Scand. 2017;96(8):939-48. doi:10.1111/aogs.13149.

24. Adedokun BO, Morhason-Bello IO, Ojengbede OA, Okonkwo NS, Kolade C. Help-seeking behavior among women currently leaking urine in Nigeria: Is it any different from the rest of the world? Patient Prefer Adherence. 2012;6:815-9. doi:10.2147/PPA.S24911.

25. Wood LN, Anger JT. Urinary incontinence in women. BMJ. 2014;349. doi:10.1136/bmj.g4531. 